INPLASY

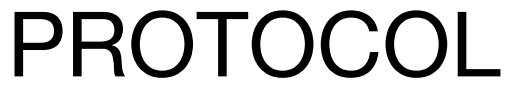

To cite: Ubaku et al.

Effectiveness of exercise in the management of motor impairments, muscle atrophy and muscle strength in PLWH: a systematic review. Inplasy protocol 2020100099. doi: 10.37766/inplasy2020.10.0099

Received: 23 October 2020

Published: 26 October 2020

Corresponding author: Collins Ubaku

ubakuprincecolin@gmail.com

Author Affiliation:

Department of Medical

Rehabilitation, University of

Nigeria, Enugu campus

Support: None.

Review Stage at time of this submission: Formal screening of search results against eligibility criteria.

Conflicts of interest:

None.

\section{Effectiveness of exercise in the management of motor impairments, muscle atrophy and muscle strength in PLWH: a systematic review}

Ubaku, C1; Ibeneme, S2; Anieto; E³.

Review question / Objective: What is the effectiveness of exercise in the management of motor impairments, muscle atrophy and muscle strength in people living with HIV/AIDS? Condition being studied: HIV infection.

Information sources: PubMed; The Cochrane Library; Pedro; MEDLINE; AMED; CINAHL; Ovid; Embase.

Study designs to be included: The review will include Randomized controlled trials (RCTs), clinical trials, crossover trials that investigated the effectiveness of exercise in the management of motor impairments, muscle atrophy and muscle strength in PLWH.

INPLASY registration number: This protocol was registered with the International Platform of Registered Systematic Review and Meta-Analysis Protocols (INPLASY) on 26 October 2020 and was last updated on 26 October 2020 (registration number INPLASY2020100099).

\section{INTRODUCTION}

Review question / Objective: What is the effectiveness of exercise in the management of motor impairments, muscle atrophy and muscle strength in people living with HIV/AIDS?

Condition being studied: HIV infection.

\section{METHODS}

Search strategy: (HIV/AIDS OR HIV OR AIDS OR RNA VIRUSES OR RETROVIRIDAE OR HIV infection OR PLWH OR PLWHA OR HIV-1 OR HIV-2) AND (Physical activity OR Exercise OR Training OR Resistance exercise OR Endurance exercise OR Aerobic exercise) AND (Motor impairment 
OR Parkinson disease OR Gait OR Ataxia OR Stroke OR Muscular dystrophy OR Muscle loss OR Apoptosis OR Aging OR Muscle function OR Muscle thickness OR Endurance OR Aerobic capacity).

Participant or population: Adult human participants aged $>=18$ years living with HIV.

Intervention: Exercise interventions which could be home-based or carried out in a clinical setting. The exercises could include aerobic exercises (e.g. bicycle ergometry, treadmill exercises,) or strengthening/ resistance exercises ( e.g weight-bearing exercise).

Comparator: Any other intervention, no intervention or routine activities.

Study designs to be included: The review will include Randomized controlled trials (RCTs), clinical trials, crossover trials that investigated the effectiveness of exercise in the management of motor impairments, muscle atrophy and muscle strength in PLWH.

Eligibility criteria: 1 . The studies must be published in English language. 2. The studies must be Randomized control trials (RCTs), they are the studies that will be included during the screening process. 3. The studies must contain the intervention and the population of the research topic.

Information sources: PubMed; The Cochrane Library; Pedro; MEDLINE; AMED; CINAHL; Ovid; Embase.

Main outcome(s): The primary outcomes of interest are motor impairments, muscle atrophy, muscle strength (e.g. measured with Oxford muscle grading).

\section{Additional outcome(s): None.}

Quality assessment / Risk of bias analysis: The PEDro scale will be used to assess the risk of bias in the included studies. The PEDro scale is a scale for appraising quality clinical trials and Bias in the included studies. The PEDro scale will be based on the Delphi list developed by Verhagen and colleagues at the Department of Epidemiology, University of Maastricht (Verhagen et al, 1998). This scale was developed by the Physiotherapy Evidence Database and consist of a checklist of 10 scored YES or NO questions pertaining to the internal validity and statistical information provided in the study. Poor quality $=\leq 3$; Fair/moderate quality $=4-5$; high quality $=6-10$. Poor quality study means that the study has a high risk of bias, while high quality study means the study have a low risk of bias. The risk of bias will be assessed by two independent reviewers. The areas of differences will be resolved by discussion and reflection, or a third reviewer will be consulted. The Appraisal of quality of included studies will be tackled after the of selection process is completed, and during the extraction and synthesis of data. The strength of Evidence for this review will be is reported.

Strategy of data synthesis: First, the question of the effectiveness of exercise would be answered, and thus all quantitative study results which examined the effect of this intervention will be presented, compared, and pooled in an evidence table. Appropriate statistical techniques will be used for each type of continuous variables (weighted mean differences if outcomes are consistent or standard mean difference if different outcomes are used, with $95 \% \mathrm{Cl}$ ) and dichotomous variables (risk ratios, with 95\% Cl). The Analysis and presentation of results will be made in hierarchical order with the outcomes coming before the additional variables. Studies will be interpreted using narrative synthesis following the recommendation of the Centre for Reviews and Dissemination to explore the relationship and findings between and within the included studies (Akers et al. 2009). A meta-analysis will be conducted for homogenous studies.

Subgroup analysis: When there is a grouped homogeneity (e.g. homogenous outcome measurement instrument, intervention type) in the included articles, a 
subgroup meta-analysis will be conducted for a grouped overall estimate of effects.

Sensibility analysis: 1. Test for heterogeneity will be conducted to determine the impact of studies with high risk of bias on the overall estimate of effect of the intervention across the included studies.

Language: English language.

Country(ies) involved: Nigeria.

Keywords: Systematic review; meta analysis; H I/A IDs infection; Musculoskeletal disease.

Contributions of each author:

Author 1 - Collins Ubaku - The author will carry out the screening, data extraction and quality appraisal.

Author 2 - Sam Ibeneme - The author provided the project topic and also supervises the research.

Author 3 - Ebuka Anieto - The author searched the various data bases. 